3E TAKANO, Toshiyuki ${ }^{1,2,3,4}\left({ }^{1}\right.$ Dept. Popul. Genet., Natl. -11 Inst. Genetics, ${ }^{2}$ Dept. Biosyst. Sci., SOKENDAI, ${ }^{3}$ Dept. Genet., SOKENDAI, ${ }^{4}$ Dept. Biol. Sci.s, Univ. of Tokyo)

An evolutionary model of cis- and trans-regulatory changes revealed by allele-specific expression analysis in hybrids

There is apparently abundant genetic variation in gene expression in natural populations. Variation is due to both cis- and trans-regulatory changes, and their relative contributions attract interests in recent years. Wittkopp et al. (2008) reported a difference in the relative contributions of cis- and transregulatory changes between intra- and inter-specific variation, suggesting a selective change or changes during evolution. However, no theoretical framework for this argument is provided. Using simulated data, I show that the contribution of transregulatory changes increases over evolutionary time without any change of selective force. I also show that the relative mutational magnitudes of cis- and trans-regulatory changes determine evolution of their relative contribution, particularly at the early stages of divergence.

3E MANO, Shuhei ${ }^{1}$, ZENG, Kai ${ }^{2}$, WU, Chung-I. ${ }^{3}\left({ }^{1} \mathrm{Grad}\right.$. -12 Sch. Natural Sci., Nagoya City Univ., ${ }^{2}$ School of Biol. Sci.s, Univ. of Edinburgh, ${ }^{3}$ Div. Biol. Sci.s, Univ. of Chicago)

Detecting positive selection based on haplotype frequency distribution

An approximate sampling formula for the infinite allele model at the end of a selective sweep is obtained, in terms of a weighted binomial mixture of the Ewens sampling formula. The approximate sampling formula is based on the hitchhiking model proposed by Maynard Smith and Haigh (1974). By using the approximate sampling formula, a new likelihood based test to detect recent selective sweep is presented. Although the test is slightly less powerful than the test based on the frequency of the most common haplotype as a summary statistics, when the size of the haplotypes cover short region. However, the test gives maximum likelihood estimates of the selection coefficient and the position of the target of selection.

\section{Workshops (W1-1 - W12-5)}

W1 FURUSE, Tamio ${ }^{1}$, WADA, Yumiko ${ }^{2}$, HATTORI, Kotaro ${ }^{3}$, -1 YAMADA, Ikuko ${ }^{1}$, KUSHIDA, Tomoko ${ }^{1}$, SHIBUKAWA Yoko $^{1}$, MASUYA, Hiroshi ${ }^{4}$, KANEDA, Hideki ${ }^{1}$, MIURA, Ikuo $^{1}$, KOBAYASHI, Kimio ${ }^{1}$, SEZUTSU, Hideki ${ }^{5}$, GONDO, Yoichi $^{6}$, SHIROISHI, Toshihiko ${ }^{7}$, YUASA, Shigeki ${ }^{3}$, WAKANA, Shigeharu ${ }^{1}$ ( ${ }^{1}$ Tech. and Dev. team for mouse phenotype analysis: Japan Mouse Clinic, RIKEN Bioresource Center, ${ }^{2}$ Dept. Welfare and Psychology, Health Sci. Univ., ${ }^{3}$ Dept. Ultrastructural Res., Natl. Inst. NeuroSci., Natl. Cent. of Neurology and Psychiatry, ${ }^{4}$ Tech. and Dev. unit for knowledge base of mouse phenotype, RIKEN Bioresource Center, ${ }^{5}$ Transgenic Silkworm Res. Cent., Natl. Inst. AgroBiol. Sci.s, ${ }^{6}$ Mutagenesis and Genomics Team, RIKEN Bioresource Center, ${ }^{7}$ Mammalian Genet. Lab., Genetic Strains Res. Cent., Natl. Inst. Genet.)

Genetic analysis and phenotype analysis of ENU induced mutant mouse that shows hyper activity

We carried out large-scale phenotype screening to establish human disease models in RIKEN ENU (N-ethyl N-nitrosourea) mutagenesis project. A hyperactive mouse, M100174, was isolated from progeny of C57BL/6J males that were treated with ENU and untreated DBA/2J. In high throughput whole genome scanning using SNPs markers, causative locus for hyperactivity was mapped to proximal region of chromosome 2. In detailed linkage analysis, the responsible mutation for hyper activity was mapped between D2Mit81 and D2Mit521. As a result of genome sequence analyses of candidate genes, a missense mutation with amino acid substitution in the C0 domain of Grin1 gene that encodes NMDA receptor subunit 1 (NMDAR1) was identified. At this meeting, we will report about following phenotype analyses of M-174 mutant mouse, general histology of brain tissue, home-cage activity test, social interaction, motor coordination, object exploration test, and pharmacological analyses with effects of methylphenidate treatment in the open-field activity, c-Fos expression pattern in brain tissue, and ERK phosphorylation level at nucleus accumbens.

\section{W1 KIMURA, Ken-Ichi ${ }^{1}\left({ }^{1}\right.$ Lab. Biol., Hokkaido Univ. of -2 Education)}

Sexually dimorphic neural circuitry: formation and function in courtship behavior of Drosophila melanogaster

The Drosophila fruitless (fru) gene product Fru has been postulated to be a neural sex determination factor. Male-specific Fru protein is expressed in small groups of neurons throughout the CNS of males, but not of females. A few tra1 homozygous, masculinized clones were generated by MARCM in the brains of female flies, the behavioral gender of which were then determined, and the masculinized cells in their brains were subsequently identified by a marker that is specifically expressed in tra1 homozygous cells. We identified a fru-expressing cell cluster, $\mathrm{P} 1$, as an important neuronal element that can initiate male-type courtship behavior. P1, located in the dorsal posterior brain, is composed of 20 interneurons and male specific. In addition, P1 expressed another sex determination factor, Doublesex (Dsx). P1 neurons are actively eliminated in the female by DsxF, the female-specific Dsx isoform. The male-specific Fru enables the male neurons to extend their neurites toward the correct targets. The male-specific formation of the P1 cluster with the ability to induce male-type courtship is secured by dual control mechanisms involving two sex-determination proteins, Fru and Dsx. 
W1 WADA, Kazuhiro ${ }^{1}\left({ }^{1}\right.$ Div. Integrated Life Sci., Faclt. -3 Adv. Life Sci., Hokkaido Univ.)

Dynamics of gene expression induced by learned vocalization

What happens in the brain during vocalization? Learned vocalization is a rare trait observed in few species, including in humans and in songbirds. In songbirds, learned singing causes increased expression of several genes in vocal nuclei. However, knowledge of behaviorally regulated genes is limited. Here, we utilized a high throughput resource, songbird 18K cDNA microarray, we developed to identify and characterize an initial cascade of vocalizing driven genes. We found over 30 genes that were regulated by singing. These genes spanned a range of functional categories to include transcription factors, repressors, chaperones, structural molecules especially associated with actin complexes, enzymes, membrane proteins. Every gene had a unique singing driven pattern in the brain, summarized in 4 main categories. Time course analysis revealed at least 6 temporal patterns. These results suggest learned vocalizing activates a dynamic motor driven cascade of different signal transduction pathways in different vocal nuclei, and that these cascades are further modified in young animals learning how to sing.
W1 HIGASHIJIMA, Shin-Ichi ${ }^{1}$, KIMURA, Yukiko $^{1}$, -5 SATOU, Chie $^{1}\left({ }^{1}\right.$ Okazaki Inst. Integ. Biosci., Natl. Institutes of Natural Sci.)

Development and function of spinal locomotor circuits in zebrafish

Recent molecular genetic studies have implicated a number of transcription factors in the specification of different interneurons. Investigating how interneurons develop and function in amniotes, however, is not trivial due to enormous complexity of their spinal circuits. In contrast, zebrafish spinal circuits are much simpler with less number of distinct classes of neurons, making it more feasible to address this issue. We have set to define the morphology and functional properties of spinal neurons that express a particular transcription factor. Methodologically, the core of our approach is to visualize transcription factor positive cells by making transgenic zebrafish that express fluorescent proteins in these cells. The transparency of embryos and larvae greatly facilitate morphological and physiological analyses of the labeled neurons. We have made a number of transgenic fish lines, including chx10, dbx1 and nkx2.2. Neurons that express these genes are mainly excitatory, and appear to regulate locomotion in zebrafish. I will present our recent data on these neurons.
W1 IINO, Yuichi ${ }^{1}$, TOMIOKA, Masahiro ${ }^{1}$, ADACHI, -4 Takeshi $^{1}$, YOSHIDA, Kazushi ${ }^{1}$, TAGAWA, Takanobu ${ }^{1}$ ( ${ }^{1}$ Dept. Biophysics and Biochem., Univ. of Tokyo)

Molecular and Cellular Mechanisms of Chemotaxis and Chemotaxis Learning in C. elegans

C. elegans shows chemotaxis to various chemicals including $\mathrm{NaCl}$. In this behavior, worms show apparently random movements but eventually reach the concentration peak of the chemical. To understand the behavioral strategy for chemotaxis, we performed quantitative analysis of $\mathrm{NaCl}$ chemotaxis and revealed two different strategies, pirouette strategy and weathervane strategy, which are employed in parallel. By laser-ablation experiments, several sensory neurons and interneurons were identified that are essential for these mechanisms of chemotaxis. Chemotaxis is also plastic. When worms are exposed to $\mathrm{NaCl}$ in the absence of bacterial food, they learn to avoid $\mathrm{NaCl}$. We have previously identified insulin-like signaling pathway as essential for this type of learning. Expression of the insulin-like peptide INS-1 in any of a small subset of neurons was found sufficient for learning. These include ASI sensory neurons known to sense pheromones, and RIC interneurons assumed to respond to starvation. INS-1 expressed in RIC was regulated by dopamine, which is known to be secreted from the CEP food-sensing neurons. Therefore INS-1 may be involved in the regulation of learning by food availability. 
WATANABE, Takaaki ${ }^{1}$, HORIUCHI, Takashi ${ }^{1}\left({ }^{1}\right.$ Div. Genome Dynamics, Natl. Inst. Basic Biology)

Development of novel inducible gene amplification systems

Gene amplification can facilitate the acquisition of a new gene function through mutations and is thought to have contributed to gene evolution. However, this concept has not been confirmed experimentally, because gene amplification in nature occurs at very low frequency and generates complicated products through several mechanisms. To gain a better understanding, we have tried to artificially induce gene amplification on a genome. We here describe gene amplification systems in budding yeast that is based on double rolling-circle replication (DRCR) utilizing two distinct reactions. These systems produced intra- and extrachromosomal products, which resemble amplified products in mammalian cells (HSR and DMs), at 100- to 1000-fold higher frequency than that in conventional systems. We are currently constructing the DRCR amplification system in mammalian cells.
W2 OHNO, Mizuki ${ }^{1}$, SAKUMI, Kunihiko ${ }^{1}$, NAKABEPPU, -3 Yusaku ${ }^{1}{ }^{1}$ Div. Neurofunctional Genomics, Dept. Immunobiology and NeuroSci., Med. Inst. Bioregulation, Kyushu Univ.)

8-Oxoguanine enhances chromosomal recombination and thus contributes genomic diversity

We have shown that a genome-wide distribution of 8-oxoguanine correlates with the preferred regions for recombination and single nucleotide polymorphism in the human genome. To obtain experimental evidences for the hypothesis that 8-oxoG is one of the main causes of frequent chromosomal recombination, we examined 8-oxoG accumulation and chromosomal recombination in mice lacking Ogg1 encoding 8-oxoG DNA glycosylase and/or Mth1 encoding 8-oxo-dGTPase. We obtained results indicating that 8-oxoG accumulated in the genome enhances chromosomal recombination in both somatic and germ cells, thus suggesting that 8-oxoG contributes to the genomic diversity in mammals.
W2 KINOSHITA, Kazuo ${ }^{1}\left({ }^{1}\right.$ Evolutionary Medicine, Shiga -2 Medical Center Res. Inst.)

Antibody and cancer evolution by an endogenous mutagen AID

Evolution occurs not only in the germline but also in somatic cells. There are two examples of somatic evolution, both of which may involve a common enzyme, activation-induced cytidine deaminase (AID). The first is the evolution of antibody gene during immune response. AID introduces massive point mutations in the immunoglobulin genes. B lymphocytes with mutations producing high-affinity antibodies are selected by antigen. AID is also required for a DNA recombination called class-switch recombination. The second is the evolution of cancer. Human epithelial cells infected with pathogens can express AID. Cancers of the stomach and liver associated with chronic infection often express AID. AID is required for a recurrent chromosomal translocation in lymphoma in a mouse model. These findings suggest that AID is involved in the evolution of cancer.

\section{W2 KODAMA, Ken-Ichi ${ }^{1}$, HORIUCHI, Takashi ${ }^{1}\left({ }^{1}\right.$ Genome -4 dynamics, NIBB)}

Micro-evolution experiments using $E$. coli K12: gaining the ability to catabolize Xylitol as a carbon source

$E$. coli $\mathrm{C}$ strain has a set of genes whose products can catabolize the two structure-isomeric sugar alcohols, Ribitol and D-Arabitol. If one of the genes, encoding Ribitol Dehydrogenase (RDH), is amplified or mutated, the $\mathrm{C}$ strain gains the ability to catabolize xylitol (Xyl), which is originally unable to be used as a carbon source. Here, using a modified $E$. coli K12 strain, into which the set of genes had been introduced from $\mathrm{C}$ strain, we investigated the relationship between the ability to catabolize Xyl and genomic changes as a model system of micro-evolution of gene. We found that the K12 strain gained the ability to utilize Xyl by (1) IS insertion or base change mutation in the repressor gene for $\mathrm{RDH}$ and (2) $\mathrm{RDH}$ gene amplification. 


\section{Environmental adaptation using plant hormone}

Habitat expansion is an important adaptational strategy of living organisms to survive unfavorable environments. However, overcoming adverse environmental conditions is not easy for plants due their sedentary nature. To overcome this constraint, plants evolve and gain new functions to fit in severely inhospitable environments and survive adverse conditions. Deepwater rice succeeded for evolved to adapt for flooding. It does not show significant internode elongation in shallow water, whereas deep water in flood induces dramatic internode elongation. I will introduce the molecular mechanism of deepwater rice.

Strategies of plants to adapt to mineral stresses in problem soils

Mineral stresses including deficiency of essential minerals and excess of essential and other minerals are the major factors limiting crop production on problem soils, which comprises $70 \%$ of world's arable land. However, some plant species or cultivars have evolved to adapt to these stress conditions. Two examples will be given on the strategies of plants to overcome $\mathrm{Al}$ toxicity on acid soils and Fe-deficiency stresses on alkaline soils. Ionic aluminum inhibits the root growth, but some Al-tolerant cultivars of barley secretes citrate from the roots to detoxify $\mathrm{Al}$ externally. We recently have identified a gene which is involved in the $\mathrm{Al}$-activated citrate secretion. We also identified a gene encoding a putative $\mathrm{ABC}$ transporter, which is required for high $\mathrm{Al}$ tolerance in rice. On the other hand, iron acquisition in barley is characterized by secretion of phytosiderophores, mugineic acids, from the roots. We have identified several genes which are involved in iron acquisition in barley and rice.

\section{W4 IMAIZUMI-ANRAKU, Haruko ${ }^{1}$ ( ${ }^{1}$ Natl. Inst. AgroBiol. -3 Sci.)}

Evolution of intracellular root symbioses with soil microbes development of strategies for nutrient acquisition in higher plants

Intracellular symbioses have been evolved as a mean of nutrient absorption in plants. Rhizobial nitrogen-fixing symbiosis and arbuscular mycorrhizal symbiosis represent mutualistic symbiosis systems in plants. More than $80 \%$ of land plants engage in mycorrhizal symbiosis, which was established 400 million years ago. It has been proposed that promotion of the phosphate uptake by mycorrhization aided plants to expand into land. While, rhizobial symbiosis arose 60 million years ago and is restricted to a number of plant species including legumes. Among leguminous mutants blocked at the early stages of rhizobial infection, some are also defective in mycorrhizal colonization. The mutated genes of the mutants compose a common signaling pathway (CSP) that controls both rhizobial and mycorrhizal endosymbioses. Thus legume-rhizobium symbiosis is thought to have the evolutional origin in preceding mycorrhizal symbiosis which is widely distributed in plant kingdom. Recent study of CSP implies that activation of CSP is prerequisite for successful infection of endosymbionts. The possible function of CSP in perception of endosymbiont infection will be discussed.

W4 HIGASHIYAMA, Tetsuya ${ }^{1,2}$ ( ${ }^{1}$ Div. Biol. Sci., Grad. -4 Sch. Sci., Nagoya Univ., ${ }^{2}$ PRESTO, JST)

Pollen Tube Attractants and Strategy of Plant Reproduction

The pollen tube attractant is a key molecule in plant reproduction. The concept of a pollen tube attractant was proposed in the late nineteenth century when pollen tubes were found to grow toward excised pistil tissues on medium. Since then, for about 140 years, plant biologists have tried to identify pollen tube attractants. However, no molecule has been convincingly demonstrated to be the true attractant that actually controls the navigation of pollen tubes in the pistil. The past decade has seen substantial progress in this field in terms of our understanding of the various mechanisms of pollen tube guidance. It was suggested that diffusible pollen tube attractants might provide localized signals that affect the directional growth of the pollen tube, especially in the last phase of guidance by the target female gametophyte. In this symposium, we discuss properties of the pollen tube attractant(s), which will provide insights into the strategy of plant reproduction. 


$$
\text { W5 TSUNEWAKI, Koichiro }{ }^{1} \text { ( }{ }^{1} \text { none) }
$$

On improvement of the infrastructure for continuous development of science in our community

Nowadays, young scientists seem to have strong wish to go abroad for the study but many of them have no strong intension to stay there for lifetime researches because of our improved scientific infrastructure. In certain priority areas, amount of fund was increased, its duration was extended and supporting stuff employment was realized. In compensation for it, ordinary research expenses supported a large number of researchers in the past tend to be reduced. It is impossible to screen researcheridea combinations for future accomplishments from many applications. A possible safety net for overcoming this problem is to support a large number of researchers with minimized selection and low cost for a long term. Second point is the problems encountered by popularization of IT. Now, IT is indispensable for research works; applying for grants, pursuing researches, and reporting the results. Researchers belonging to established organizations are benefited by the institutional IT system, but researchers not associated with such organizations are handicapped in receiving the benefit from IT. Our Society needs to pay some attention to this problem.

\section{W5 MORIWAKI, Kazuo ${ }^{1}\left({ }^{1}\right.$ RIKEN Tsukuba Institute, Bio- -2 Resource Center)}

Bioresource in the Genome Age

Extensive analysis of genome structures in man and other organisms has provided a powerful tool for the modern life science research. At the same time, we have to step into the complex architecture involved in genetic regulation of biological functions of living individuals. In this century, we again need forward genetics methodology starting from individual phenotypes, together with the reverse genetics approach. To meet this trend, it is necessary to establish bioresources with higher genetical and microbiological quality which are effective in advanced molecular analysis. Western countries have already established bioresources with higher quality as the essential basis for advanced life science research. Japan also started the National Bioresource Project in 2001, although its sustainability is still unclear. Further understanding and support of this bioresource project by our research community is strongly desired. Furthermore, the use of bioresources supplemented with their history of establishment and also with the evolutionary history of the species as the progenitor of each resource, should be advantageous for our original research in the future.

\section{W5 SEKIGUCHI, Mutuo ${ }^{1}\left({ }^{1}\right.$ Fukuoka Dental College)

History of gene analysis and Genetic Society of Japan

The molecular analyses of gene function and signal transduction had established in the latter half of the 20th century. What is the goal of molecular genetics in the near future? What kind of works are necessary in the function of the Genetic Society of Japan? Several possibilities are proposed on these questions. 
TAKAO, Kondo ${ }^{1}$, KITAYAMA, Yoko ${ }^{1}\left({ }^{1}\right.$ Div. Biol. Sci Grad. Sch. Sci. Nagoya Univ.)

The multiple coupled oscillations, based on KaiC, constitute the circadian clock of cyanobacteria

Circadian rhythms, biological oscillations of physiological activities with a period of $\sim 24 \mathrm{~h}$, are found in a wide spectrum of organisms and enhance their fitness in a day/night cycle. Cyanobacteria are the simplest organisms known to exhibit circadian rhythms. In the cyanobacteria, the KaiA, KaiB, and KaiC proteins are essential proteins for circadian rhythm generation. The autonomous circadian cycling of KaiC phosphorylation is thought to be the basic pacemaker of the circadian clock. In this study, we investigated the rhythms in a kaiAoverexpressing strain, in which the cycle of KaiC phosphorylation was impaired, and found that there were circadian rhythms of gene expression. We also found that circadian rhythms were maintained at low temperature only when both the KaiC phosphorylation cycle and the transcription and translation cycle were intact. Thus, we propose that multiple coupled-oscillatory systems based on the biochemical properties of KaiC are important to maintain robust and precise circadian rhythms in cyanobacteria.
W6 YAMASHINO, Takafumi ${ }^{1}$, MIZUNO, Takeshi ${ }^{1}\left({ }^{1}\right.$ Lab. -3 Molec. MicroBiol., Sch. Agr., Nagoya Univ.)

Genetic studies provide us with a molecular picture of the plant circadian clock

During the last decade, we have seen a remarkable advance in our understanding of the plant circadian system. In Arabidopsis thaliana, a number of clock-associated genes have been uncovered, the products of which appear to be implicated in the central clock system that generates rhythmic oscillations in cellular and physiological activities of plants. We have been characterizing one core group of Arabidopsis clock components that is believed to control the pace of the central oscillator. They are five members of the PSEUDO-RESPONSE REGULATOR (PRR) family, including TIMMING OF CAB2 EXPRESSION 1 (TOC1/PRR1). We established a complete set of multiple prr mutants, including a prr 9 prr7 prr5 toc1 quadruple mutant, and then extensive genetic studies were conducted with reference to their clock-associated phenotypes, including the photoperiodic control of flowering time and the photo-sensitivity of plant growth. Based on these recent genetic data, we will discuss the molecular picture of the Arabidopsis circadian clock, in which the PRR members coordinately play essential roles.
W6 MATSUO, Takuya ${ }^{1}$, OKAMOTO, Kazuhisa ${ }^{1}$, ONAI, -2 Kiyoshi ${ }^{1}$, NIWA, Yoshimi ${ }^{1}$, SHIMOGAWARA, Kosuke ${ }^{2}$, ISHIURA, Masahiro ${ }^{1}\left({ }^{1}\right.$ Cent. Gene Res., Univ. of Nagoya, ${ }^{2}$ Lab. Chemistry, Sch. Med., Univ. of Teikyo)

The circadian clock of green algae: Comprehensive identification of circadian clock genes in Chlamydomonas reinhardtii

The unicellular green alga Chlamydomonas reinhardtii have been used to study circadian rhythms, but their clock genes remain to be identified. Since yeasts do not exhibit circadian rhythms, Chlamydomonas, referred as "Green yeast", would be the simplest eukaryotic model system for circadian rhythm research. To establish it as a new model for studying molecular mechanisms of the circadian clock, we comprehensively identified clock genes by a highly efficient forward genetic analysis. In this workshop, we would like to introduce the method and results of the analysis, and the features of circadian clock of Chlamydomonas.
W6 YOSHIMURA, Takashi ${ }^{1}\left({ }^{1}\right.$ Lab. Animal Physiology, -4 Grad. Sch. Bioagricultural Sci., Nagoya Univ., ${ }^{2}$ Avian Bioscience Res. Cent., Grad. Sch. Bioagricultural Sci., Nagoya Univ.)

\section{Functional genomics of seasonal time measurement}

Animals living outside the tropics use changes in day length to adapt to seasonal changes in environment, but the molecular mechanisms underlying seasonal time measurement are not fully understood. The Japanese quail is a robust model for the study of these mechanisms because of its rapid and dramatic response to changes in photoperiod. To address the photoperiodic signal transduction pathway we have dissected the molecular dynamics of gene expression using a chicken high density oligonucleotide microarray. Two waves of gene expression were identified. The first was initiated about $14 \mathrm{~h}$ after dawn of the first long day and included increased thyrotropin (TSH) $\beta$ subunit expression in the pars tuberalis of the pituitary gland. The second occurred about 4 $\mathrm{h}$ later and included increased type 2 deiodinase (DIO2) expression in the ependymal cell (EC) lining ventrolateral walls of the third ventricle. TSH receptor was found in the EC and ICV administration of TSH to short day quail stimulated gonadal growth, and expression of DIO2 in the EC. Increased pars tuberalis TSH therefore appears to trigger long day photoinduced seasonal breeding. 
W6 EBIHARA, Shizufumi ${ }^{1}$, TOMIDA, Shigeru ${ }^{1}$, MAMIYA, -5 Takayoshi ${ }^{3}$, SAKAMAKI, Hirotake ${ }^{1}$, KAMEYAMA, Tsutomu ${ }^{4}$, NABESHIMA, Toshitaka ${ }^{3}$, KOBAYASHI, Junya $^{1}$, IWAKI, Yuka ${ }^{1}$, IMAI, Saki ${ }^{1}$, ISHIKAWA, Akira $^{2}$, ABE, Kuniya ${ }^{5}$, YOSHIMURA, Takashi ${ }^{2}{ }^{1}$ Div. Biomodeling, Grad. Sch. Bioagricultural Sci., Nagoya Univ., ${ }^{2}$ Div. Applied Genetics and Physiology, Grad. Sch. Bioagricultural Sci., Nagoya Univ., ${ }^{3}$ Dept. Chem. Pharmacology, Faclt. Pharmacy, Meijo Univ., ${ }^{4}$ Japan Institute of Psychopharmacology, ${ }^{5}$ RIKEN BioResource Center)

Identification of a quantitative trait gene underlying behavioral despair using CS mice with abnormal circadian rhythms

The tail suspension test (TST) and the forced swimming test (FST) are widely used experimental paradigms for assessing antidepressant activity and depression-like behavior. We found that CS mice exhibit virtually no immobile posture when placed in inescapable situations. Quantitative trait locus (QTL) mapping using CS and C57BL/6J mice detected significant QTLs in the TST and FST on chromosome (Chr) 5 and in the FST on Chr 4. To identify the quantitative trait gene located on $\mathrm{Chr} 5$, we narrowed the critical chromosomal region with a number of congenic and sub-congenic strains. In the narrowed chromosomal region, we found one coding mutation. This mutation affects other behaviors including nest building, alcohol preference, wheel-running behavior, and righting reflex induced by muscimol. Transgenic mice with BAC containing the wild-type gene completely rescued all these phenotypes. These results indicate that this gene affects TST and FST immobility and suggest that the gene is implicated in the regulation of the action of GABA.
W7 SATO, Yutaka ${ }^{1}\left({ }^{1}\right.$ Grad. Sch. Bioagricultural Sci., -1 Nagoya Univ.)

Molecular mechanism of transposons escaping from host's silencing machinery and its contribution to rice genome evolution

Transposons are genomic parasites, which constitute a major component of eukaryotic genome. RNA silencing machinery is a defense system against them, and suppresses transposons to stabilize host's genome. It is a nature of transposons as a selfish DNA element to increase their copies, despite the presence of this defense system. This implies the existence of a pathway, which enables transposons to escape host's defense. I am analyzing one miRNA, miRJ, whose target is de novo DNA methyltransferase. miRJ is originated from recently produced inverted repeats of a part of a gene encoding de novo DNA methyltransferase. Interestingly, these inverted repeats seem to locate within the CACTA DNA transposons dispersed at 5 loci in rice genome. This finding evokes a model how transposons escape from silencing machinery of host. In the workshop, I will discuss a possible mechanism of transposons escaping from host's silencing and its contribution to genome evolution in rice.

W7 SIOMI, Haruhiko ${ }^{1}\left({ }^{1}\right.$ Dept. Molec. Biol., Keio Univ. -2 Sch. Med.)

A link between RNAi and retrotransposon silencing

RNAi is a conserved mechanism in which small RNAs trigger various forms of sequence specific gene silencing by guiding Argonaute complexes to target RNAs via base-pairing. In Drosophila, there are five separate members of the Argonaute gene family, which can be subdivided according to sequence and expression pattern into two subfamilies: the AGO (AGO1 and AGO2) and PIWI (Aubergine, Piwi, and AGO3) subfamilies. piRNAs with 24-28 nucleotides in length arise from repetitive intergenic elements including retrotransposons by a Dicerindependent pathway in the Drosophila germline and function through the PIWI subfamily to ensure silencing of retrotransposons. In Drosophila cultured somatic S2 cells, AGO2, the AGO subfamily member, associates with endogenous small RNAs with 20-22 nucleotides in length, which we have collectively named endogenous siRNAs (esiRNAs). esiRNAs mainly correspond to a subset of retrotransposons. esiRNAs are produced in a Dicer-2dependent manner and can direct AGO2 to cleave target RNAs. Together, these findings indicate that in Drosophila, different types of small RNAs and Argonautes are utilized to repress retrotransposons in germline and somatic cells. 
W7 SASAKI, Hiroyuki ${ }^{1,2}$, WATANABE, Toshiaki ${ }^{1,2}\left({ }^{1}\right.$ Div -3 Human Genet., Natl. Inst. Genet., Research Org. Inform. and Systems, ${ }^{2}$ Dept. Genet., Sch. Life Sci., Grad. Univ. Adv. Stud.)

Endogenous siRNAs derived from naturally formed dsRNAs regulate coding-transcripts and retrotransposons in mouse oocytes

RNA interference (RNAi) is a mechanism by which doublestranded RNAs (dsRNAs) regulate transcripts in a sequencedependent manner. dsRNAs are processed by Dicer to short interfering RNAs (siRNAs), which are then incorporated into Argonaute (Ago) proteins. In mammals, where no RNA-dependent RNA polymerase activity has been found, biogenesis and function of endogenous siRNAs remain unknown. By means of deep sequencing, however, we identify a large number of both 2527-nt Piwi-interacting RNAs (piRNAs) and 21-nt siRNAs corresponding to mRNAs or retrotransposons in growing oocytes. piRNAs are bound to Mili and have a role in regulation of retrotransposons. Endogenous siRNAs are exclusively mapped to retrotransposons or other genomic regions that produce transcripts capable of forming dsRNAs. Some siRNAs are derived from expressed pseudogenes, indicating that one role of pseudogenes is to regulate homologous mRNAs through RNAi. Loss of Dicer or Ago2 results in decreased levels of siRNAs and increased levels of target transcripts. The findings suggest that the canonical RNAi pathway is highly conserved through evolution.

W7

OKADA, Norihiro ${ }^{1}{ }^{1}$ Tokyo Inst. of Tech., Faclt. of -4 Biosci. \& Biotech.)

Mammalian-specific brain was made by insertions of retroposons?

In spite of many cases of retroposons acquiring function during evolution, no cases are reported in which retroposon insertions are involved in creation of a new morphological innovation specific to a certain taxonomic group. We characterized previously around 100 copies of a SINE family, named AmnSINE1, present as a part of conserved non-coding elements, CNEs, in mammalian genomes, proposing a hypothesis that they have acquired genomic functionality in a common ancestor of mammals to accomplish characteristics specific to mammals. Here using a mouse enhancer assay, we clearly demonstrated that one locus functions as an enhancer specific to the expression of Fgf8 gene in the two forebrain regions. We also demonstrated that the region is responsible for patterning of barrel structure in thalamus. We also discovered that another locus named AS021 functions as an enhancer for Satb2, which directs the expression in a neocortex layer. We hypothesize here that these two enhancers play essential roles in the brain development as a part of the huge mammalian-specific neuronal network created newly by the exaptation of AmnSINE1 in a common ancestor of mammals.
W8 TAKAHASHI, K. ${ }^{1}$, TAJIMA, F. ${ }^{2}\left({ }^{1}\right.$ Inst. Genet., ${ }^{2}$ Dept. -1 Biol. Sci.s, Grad. Sch. Sci., Univ. Tokyo)

Effect of drastic population contraction on the transient dynamics of mean fitness

A large, equilibrium population suffers from a reduction in mean fitness through the balance between recurrent deleterious mutation and purging natural selection. We here show that when the population undergoes a sudden decline in its size, the mean fitness may be decreased even more substantially. By numerically analyzing a Fisher-Wright transition matrix model with varying population size, we find that this reduction in mean fitness becomes more evident when [i] deleterious alleles are more recessive, or [ii] the change in population size is more drastic. Soon after the contraction, however, the mean fitness quickly goes back to its equilibrium level, suggesting that the deteriorating effect of drastic population contraction is only transient.
W8 MANO, Shuhei ${ }^{1}\left({ }^{1}\right.$ Grad. Sch. Natural Sci., Nagoya -2 City Univ.)

Ancestral process and diffusion model with selection

The ancestral selection graph (Krone \& Neuhauser, 1997) is an analogue to the coalescent genealogy. The number of ancestral particles, backward in time, of a sample of genes is an ancestral process, which is a birth and death process with quadratic death and linear birth rate. An explicit form of the number of ancestral particle is obtained, by using the density of the allele frequency in a diffusion model obtained by Kimura (1955). It is shown that fixation is convergence of the ancestral process to the stationary measure. The time to fixation of an allele is studied in terms of the ancestral process. 
W8 TESHIMA, Kosuke ${ }^{1}\left({ }^{1}\right.$ Hayama Center for Adv. -3 Studies, The Grad. Univ. for Adv. Studies)

Evolutionary process of duplicated genes under the pressure of gene conversion

The evolutionary process of duplicated genes are studied. The process is very complicated because of the effect of gene conversion. At first, our simulation study demonstrated the duplicated genes experience a very long phase of concerted evolution. Next, we considered neofunctionalization process. Assume a neofunctionalization mutation arises on one of the duplicated genes. This newly arisen allele is maintained by the combined effect of selection and gene conversion. As a result of the balance of these factors, it was found that a distinctive pattern of the spacial distribution of the divergence is generated. The spacial distribution likely has a peak around the target site of selection. Because this peak is limited to the local region, however, it is easy to overlook unless carefully analyzed. The result of the theoretical research was applied to the human redand green-opsin genes. The theoretical prediction explains well when we assume selection works on the two amino acid differences between two opsins.
W9 HOSHINO, Atsushi ${ }^{1}$, MORITA, Yasumasa ${ }^{1}$, PARK, $-1 \quad$ Kyeung-Il ${ }^{1}$, CHOI, Jeong-Doo ${ }^{1}$, IIDA, Shigeru ${ }^{1}\left({ }^{1}\right.$ Natl. Inst. Basic Biol.)

\section{Epigenetics of flower variegation}

Phenotypic variations are often caused by epigenetic changes in gene expression. The recessive mutations duskish in the Japanese morning glory (Ipomoea nil) and pearly- $v$ in the morning glory (I. tricolor), conferring flower variegations, are caused by the insertions of DNA transposons of the CACTA and $M u$ superfamilies within the promoter regions of the UFGT and $D F R$ genes for anthocyanin pigmentation, respectively. The flower variegations are not associated with the excision of the transposons but are due to epigenetic changes. Indeed, DNA methylation at a particular site of the $D F R$ promoter in the pearly- $v$ mutant is associated with the suppression of $D F R$ gene expression, and the DNA methylation at the particular site appears to be affected by the methylation of the inserted $M u$ element. Consequently, somatic changes in the methylation state of the particular site cause flower variegation. We will also discuss possible mechanisms for the duskish variations.
W8 INNAN, Hideki ${ }^{1}\left({ }^{1}\right.$ Graduate Univ. for Advanced -4 Studies)

Two-locus models with interaction between loci and their applications

Various two-locus models when there is some kind of interaction between loci are introduced. The possible interactions include recombination, gene conversion and selection. We consider how to apply the analytical results of those two-locus models to real data
W9 SAZE, Hidetoshi ${ }^{1}$, SHIRAISHI, Akiko ${ }^{1}$, MIURA, -2 Asuka ${ }^{1}$, KAKUTANI, Tetsuji ${ }^{1}\left({ }^{1}\right.$ Div. Agr. Genetics, Natl. Inst. Genetics)

DNA Hypermethylation Mutant in Arabidopsis thaliana

Differential cytosine methylation of repeats and genes is important for coordination of genome stability and proper gene expression. In Arabidopsis, DNA methylation at repeated heterochromatic loci is regulated by DNA methyltransferases and a chromatin remodeling factor DDM1. Although DDM1 controls both CG and non-CG methylation, loss of DDM1 not only causes global hypomethylation but also induces local DNA hypermethylation at several genic regions, including $B N S$ (BONSAI) locus. Through a genetic screen, we identified a novel jmjC-domain containing gene IBM1 (increase in bonsai methylation1) that negatively regulates cytosine methylation at the $B N S$ genic region. $i b m 1$ induces ectopic cytosine methylation at $B N S$ and developmental phenotypes, which are due to ectopic deposition of heterochromatic H3K9 methylation mark. Our results demonstrate the importance of chromatin remodeling and histone modifications in the differential epigenetic control of repeats and genes. 
W9 IKEDA, Yoko ${ }^{1}$, KINOSHITA, Yuki ${ }^{1}$, KAKUTANI, -3 Tetsuji ${ }^{2}$, KINOSHITA, Tetsu ${ }^{1}\left({ }^{1} \mathrm{Grad}\right.$. School of Biol. Sci., ${ }^{2}$ Dept. Integrated Genetics, Natl. Inst. Genet.)

Control of genomic imprinting by $A L A R M C L O C K 1$ gene in Arabidopsis

Genomic imprinting refers to the unequal expression of maternal and paternal alleles of a gene depending on the parent-of-origin, and is a phenomenon that has evolved in both placental mammals and flowering plants. In Arabidopsis, imprinting of the maternally expressed genes MEDEA, FIS2 and FWA is established in the maternal central cell, which is the progenitor cell of endosperm before fertilization, by the activity of DEMETER, a DNA demethylase gene, while the male gametophyte allele is held silent. This one-way activation mechanism of genomic imprinting is unique to flowering plants. A process of imprinting likely begins with the recognition of imprinted genes, followed by DNA demethylation and remodeling of silent chromatin to the active state. To gain insight into this DNA demethylation and hence establishment of genomic imprinting, we have carried out genetic screening using Arabidopsis plant. We have isolated alac (alarm clock for FWA imprinting) mutant that is defective in FWA-GFP expression, and found that the gene controls all known maternally expressed imprinted genes in the central cell and the endosperm.

NASUDA, Shuhei ${ }^{1}\left({ }^{1}\right.$ Lab. Plant Genet., Grad. Sch. -4 Agr., Kyoto Univ.)

Are plant centromeres under epigenetic control? -An analysis of aberrant wheat and barley chromosomes-

Plant centromeres are large in size and complex in their constitutions. Common to the plant centromeres is that the centromeric regions are rich in satellite repeats and centromerespecific retrotransposons. Functional importance of barley centromeric repeats, namely '(AGGGAG)n satellite' and 'cereba retrotransposon', was demonstrated by chromatin immunoprecipitation with the CENH3 antibody. Barley chromosomes introduced to wheat background is a good material to study plant centromere structures, because they are dispensable and can be easily identified by cytological techniques. We induced structural aberration to the barley chromosome $7 \mathrm{H}$. We found a wheat chromosome carrying barley centromere sequences at the tip of the chromosome. The barley sequences no longer have centromeric function. We obtained a pseudodicentric chromosome that carries both wheat and barley centromeric repeats. In this case the wheat centromere lost its function. We also found barley telosomes without centromeric repetitive sequences. The peculiar feature of the activated/inactivated centromeres of the above mentioned chromosomes might be explained by the epigenetic control of plant centromere functions.
W9 NAGAKI, Kiyotaka ${ }^{1}$, KASHIHARA, Kazunari ${ }^{1}$, -5 WAKIMOTO, Munenori ${ }^{1}$, TERADA, Kaori ${ }^{1}$, YOKOTA, Etsuko ${ }^{1}$, MURATA, Minoru ${ }^{1}$ ( ${ }^{1}$ Res. Inst. for bioresources, Okayama Univ.)

Epigenetics in centromeres

Centromeres of higher eukaryotes possess centromeric DNAs, and a large number of centromere specific proteins are recruited at the centromeric DNAs for functioning the centromeres. However, positions of centromeres are not defined by the centromeric DNAs themselves, and the centromere proteins define the positions epigenetically. Among the proteins, the centromere-specific histone H3 (CENH3) locates only in functional centromeres, playing as a base for recruiting of other centromere proteins. We are now investigating effect of qualitatively and quantitatively changed CENH3 for centromere functions in plants. I discuss about the epigenetical modifications of the centromeres in this workshop. 
W10 AOKI, Kenichi ${ }^{1}$ ( ${ }^{1}$ Dept. Biol. Sci.s, Univ. of Tokyo)

$-1$

Properties of human learning and culture as predicted from evolutionary theory

Temporal and/or spatial environmental variation strongly affects the evolution of social and individual learning. In particular, individual learning is favored when large changes in the environment occur frequently and repeatedly. Of special interest is the possibility that humans repeatedly experienced large changes of the environment during their rapid range expansion from Africa. I analyze the evolution of social and individual learning in an environmentally heterogeneous stepping stone model.

W10 INOUE-MURAYAMA, Miho ${ }^{1}$ ( ${ }^{1}$ Wildlife Res. Inst. of -2 Kyoto Univ.)

Genetic polymorphism as a background of animal behavior

Various studies have shown the associations between differences in human behavioral traits and genetic polymorphism of neurotransmitter-related proteins such as receptor, transporter and monoamine oxidase. To clarify the genetic background of animal behavior, we analyzed corresponding regions in animals. In primates the polymorphism was discovered between or within species, and the functional effect on neural transmission was found to be different by alleles. In dogs allele distributions of several genes were different among breeds showing different behavioral traits, and genes associating individual difference in aggressiveness and aptitude of working dogs were surveyed. The marker genes for behavior will provide useful information for human evolution, welfare of zoo animals, and effective selection of working dogs and industry animals.
W10 ONO, Katsuhiko ${ }^{1}\left({ }^{1}\right.$ Div. NeuroBiol. and Bioinform, -3 Natl. Inst. Physiological Sci.)

Involvement of glial cells in the brain formation and its function

The central nervous system is composed of neurons and glial cells. While neurons are usually focused on to study brain function, recent evidence suggests that macroglial cells are also involved in brain function directly. In addition, the glial cell ratio in the neocortex is higher in primate than in rodent, also suggesting glial cells are important for cortical function. To understand involvement of glial cells in the brain development and function, we examined macroglial cell formation and architecture in the neocortex from phylogenical and ontogenical views. First, we study glial cell development in a phylogenical view point, and examine neuron-to-glial cell ratio in the neocortex of several mammalian species, including human brain. Second, factor(s) that determine glial cell character and its number is examined in the developing mouse forebrain. Currently, we are obtaining candidates of such factors. Based on the results, I will discuss possible glial implication in brain development. 
W11 OZAKI, Shogo ${ }^{1}$, KATAYAMA, Tsutomu ${ }^{1} \quad{ }^{1}$ Dept -1 Molec. Biol., Grad. Sch. Pharm. Sci., Kyushu Univ.)

Analysis on the minimal functional elements of the replication origin in duplex DNA unwinding by DnaA

$E$. coli DnaA binds to specific sequences (DnaA boxes) within the replication origin (oriC), which results in formation of a multimeric complex on oriC. DnaA tightly binds also ATP and ADP. Unlike ADP-DnaA, ATP-DnaA causes duplex DNA unwinding within the specific AT-rich region (DUE) of oriC. This conformational change triggers a series of reactions that lead to the loading of replicative DNA polymerase. To understand the universal mechanism of DUE unwinding, we previously analyzed initiation complexes formed by DnaA homolog (tmaDnaA) of a hyperthermophile $T$. maritima, one of the most ancient eubacterial species and found that tmaDnaA causes local unwinding of 149-bp oriC region of this bacterium in vitro in an ATPdependent manner. Here, we further investigated the minimal functional elements that is crucial for DUE unwinding by tmaDnaA. We constructed and analyzed different kinds of mutations in oriC. We found using an in vitro reconstituted system that formation of active complexes for DUE unwinding requires at least four DnaA boxes. Based on this and other results, we would like to suggest an evolutionary original structure of initiation complex.

W11 OKI, Masaya ${ }^{1}$, HATANAKA, Akira ${ }^{1}$, NOGAMI, Emi ${ }^{1}$, -2 UCHIDA, Hiroyuki ${ }^{1}\left({ }^{1}\right.$ Dept. Applied Chemistry \& Biotec., Faclt. Eng., Univ. of Fukui)

Analysis of native heterochromatin barrier function

Transcriptionally active and inactive domains are frequently found adjacent to one another in the eukaryotic nucleus. To better understand the underlying mechanisms by which domains maintain opposing transcription patterns, we performed a systematic genome wide screen for proteins that could block the spread of silencing in yeast. Using a standard silencer blocking assay we have screened all 6000 yeast genes for barrier activity when recruited to a barrier. We have identified 55 strong blockers. We also analyzed native barrier at HMR hterochromatin locus. We have already reported mechanism of right boundary, it made two overlapping mechanisms function, one of which involves a DNA binding element, while the second mechanism involves specific chromatin modifying activities which function in competition with repressors to block the spread of silenced chromatin. On the other hand, there are two Ty-LTR (Long Terminal Repeat) at left boundary region. Our data suggest that these elements are important to make barrier. In this meeting, we report a detail molecular analysis of isolated barrier proteins by a genome wide screening and native barrier function at HMR heterochromatin locus.
W11 SATORU, Ide ${ }^{1}$, HISAJI, Maki ${ }^{2}$, TAKEHIKO, -3 Kobayashi ${ }^{1,3}$ ( ${ }^{1}$ Div. Cytogenetics, Natl. Inst. Genetics, ${ }^{2}$ Dept. Molec. Biol., Nara Inst. of Sci. and Tech., ${ }^{3}$ Dept. Genet., Sch. Life Sci., The Grad. Univ. Adv. Stud. (SOKENDAI))

Extra coding functions of ribosormal RNA genes

The ribosormal RNA gene (rDNA) is one of the most abundant genes in eukaryotic cells. The budding yeast has about $150 \mathrm{rDNA}$ copies on the chromosome. However, interestingly, it is known that half of the copies are not transcribed (herein called extracopies of rDNA). In addition, as we described previously, a strain that has only 40 rDNA copies without extra-copies of rDNA can survive and grow normally, suggesting that the rDNA has functions other than production of rRNA. To test the roles of such extra-copies of rDNA, we established strains that have different rDNA copies, from 20 to 150 , and analyzed them from several aspects. As a result, we found that the low rDNA-copy strains were sensitive to DNA damage. Furthermore, our study revealed that the recombination repair using sister chromatid in the rDNA was compromised in the low copy strains by losing the cohesion. Finally, the cohesion loss in the low copy strains was caused by changing the extra-copies from the inactive mode of transcription to the active mode of that. These data suggest that the extra-copies of rDNA play a role in the maintenance of rDNA repeat by generating cohesion for recombination repair.

W11 OHTA, Kunihiro ${ }^{1}\left({ }^{1}\right.$ Dept. of Life Sci., Grad. Sch. Art \& -4 Sciences, Univ. of Tokyo)

DNA transaction in the initiation of meiotic recombination

Meiotic recombination is an important process for gamete formation and acquisition of genetic diversity. The initiation process of meiotic recombination is triggered by the Spo11mediated formation of transient DNA double strand breaks (DSBs) at recombination hotspots. Recent studies have revealed that Spo11-mediated DSB formation is under the integrated regulation systems that involve alteration of local chromatin structure, histone modification, binding of Spo11 to chromosomes, sister chromatid cohesion protein Rec8, cycle regulator Cdc7 kinase, and domain structure of chromosomes. We will overview of the current status of this field with our recent observations. 
W11 IWASAKI, Hiroshi ${ }^{1}\left({ }^{1}\right.$ International Grad. Sch. Arts -5 and Sciences, Yokohama City Univ.)

Molecular mechanism of DNA strand exchange during homologous recombiantion

DNA strand exchange reaction is a fundamental chemical reaction for homologous recombination and recombinational repair, which is mediated by so-called recombinases. Recombinases bind to single-stranded DNA, leading to formation of a helical presynaptic filament that promotes homology search and homologous pairing of a complementary DNA strand. Our genetic and biochemical studies have demonstrated that Swi5-Sfr1 protein complex from fission yeast is an activator and stabilizer of Rhp51 recombinase. Based on our last results, I will discuss about the molecular mechanism of Rhp51-Swi5-Sfr1-mdeiated DNA strand exchange in this workshop.

W11 JOHAUKA-HISATOMI, Yasuyo ${ }^{1}$, IIDA, Shigeru ${ }^{1}$ -6 ( ${ }^{1}$ Div. Molec. Genetics, Natl. Inst. Basic Biol.)

Efficient transfer of base changes from a vector to the genome by homologous recombination in rice gene targeting: involvement of heteroduplex formation and mismatch correction

Through a gene-targeting procedure with positive-negative selection, we previously reported the generation of fertile transgenic rice plants with a positive marker inserted into the $A d h 2$ gene by using an Agrobacterium-mediated transformation vector containing the positive marker flanked by two $6-\mathrm{kb}$ homologous segments for recombination. Base changes within the homologous segments in the vector were efficiently transferred into the corresponding genomic sequences of rice recombinants, and heteroduplex formation and subsequent mismatch corrections appeared to occur. Several alternative models, including the occurrence of the mismatch correction of heteroduplex molecules formed between the genomic DNA and either a single-stranded or double-stranded T-DNA intermediate, are compared to explain the observation, since a single-stranded T-DNA molecule in Agrobacterium-mediated transformation is imported into the plant nucleus and becomes double-stranded. We will also discuss implications for the modification of endogenous genes for functional genomic analysis by gene targeting.

\section{W12}

$-1$
W12 KAMADA, Hiroshi ${ }^{1}\left({ }^{1}\right.$ Gene Res. Cent., Grad. Sch. Life
-2 and Environmental Sci.s, Univ. of Tsukuba)

Safety assessment and legal regulation of genetically modified plants

In the early 1980s, transformation methods of higher plants using Agrobacterium had been developed. In Japan, guideline for recombinant DNA experiments to produce and cultivate transgenic plants had been established in the middle of $1980 \mathrm{~s}$. After several modifications of the guideline, Cartagena Law was legislated in 2004 and then, governmental assessment on environmental effects of GM plants prior for the field trial cultivation was obligated legally. On the other hand, in the $1990 \mathrm{~s}$, production of various GM crops and its utilization as foods progressed quickly and then, the guideline for food safety assessment of GM crops was established. In the early 2000s, food safety assessment of GM crops was obligated by the Food Sanitation Law and by the Food Safety Basic Law. In this presentation, I would like to explain the main content of the law and the basic concept for the production and cultivation of GM plants and for the food safety assessment of GM crops. 


\section{W12 OBATA, Yuichi ${ }^{1}\left({ }^{1}\right.$ RIKEN BioResource Center $)$ $-3$}

Derivation of genetically manipulated animals and animal expriments

Manipulation of genes in vivo has become an essential tool for elucidation of the function of genes involved in sophisticated biological systems such as development and differentiation, brain and neural functions and immune responses. Although the ultimate goal is to understand the function of genes in human health and diseases, manipulation of genes directly in human is impossible because of the ethical issues. Thus, various model animals including mouse, medaka fish, drosophila and C. elegans are valuable and irreplaceable for genetic manipulation. The most outstanding model animal is the mouse. Technologies for derivation of transgenic, knock-out and knock-in mice and for the tissue specific manipulation of target genes have been well established. Furthermore, genome-wide knockout mouse projects are in progress in Europe and North America. These studies are expected to bring numerous fruitful results. To continue these studies, however, scientists involved should gain public support and understanding by proper safekeeping and secure prevention of escape and diffusion of these genetically manipulated animals and by proper conduct of animal experiments.

W12 TAKEUCHI, Kaoru ${ }^{1}{ }^{1}$ Inst. Basic Med. Sci.s, Grad. -4 Sch. Comprehensive Human Sci.s, Univ. of Tsukuba)

Important points in virus research using recombinant DNA technology

Cartagena Protocol on Biosafety to the Convention on Biological Diversity seeks to protect biological diversity from potential risks posed by living modified organisms (LMOs) resulting from modern biotechnology. This protocol was ratified in Japan after establishing domestic law and regulations for the protocol. In the domestic law, use of LMOs is classified into type 1 use (use without containment measures) and type 2 use (use with containment measures). According to the domestic law, most of experiments using recombinant viruses are required for the approval of the Minister. In this workshop, I will explain Cartagena Protocol and the Japanese domestic low and point out important points in virus research using recombinant DNA technology.
W12 TAKEBE, Hiraku ${ }^{1} \quad\left({ }^{1}\right.$ Genetic Counseling Course, -5 Kindai Univ. Grad. Sch.)

Bioethical principle of gene analysis

For human gene analysis, ethical aspects are indispensable and important. The Government of Japan issued guidelines for the ethical aspects for human gene studies, mainly for basic research, in 2001, and revised them in 2005. Some researchers suspected the guideline as blocking the freedom of research, but such guidelines have been well accepted in many advanced countries as protection from criticism for possible lack of ethical consideration. The guidelines, both domestic and international, aim to protect the right of human subjects or patients from ethically inadequate handling of the materials obtained from them. There are considerable differences between international and domestic guidelines, mainly because, I suspect, understanding human genetics has been far behind in Japan. This is due to the lack of education of human genetics in most of medical schools in Japan. Consequently, ethical guidelines in Japan are considerably different, in principle, from international standards particularly for the need of sharing genetic information among blood relatives. 